\title{
Ecologia reprodutiva de Hydromedusa tecifera (Testudines: Chelidae) no sul do Brasil
}

\author{
Camila Kurzmann Fagundes ${ }^{1}$ \& Alex Bager ${ }^{2,3}$
}

Biota Neotropica $v 7$ (n2) - http://www.biotaneotropica.org.br/v7n2/pt/abstract?article +bn04807022007

\author{
Recebido em 22/02/07 \\ Versão Reformulada recebida em 26/06/07 \\ Publicado em 31/07/07 \\ ${ }^{1}$ Programa de Pós-Graduação em Biodiversidade Animal, \\ Universidade Federal de Santa Maria - UFSM, \\ Rua Marcelino Debacco, 78, CEP 898802-290, Santo Ângelo, RS, Brasil \\ ${ }^{2}$ Laboratório de Manejo e Conservação Ambiental, Universidade Estadual do Rio Grande do Sul, \\ Av. Assis Brasil, 842, CEP 95400-000, São Francisco de Paula, RS, Brasil \\ ${ }^{3}$ Autor para correspondência: Alex Bager,e-mail: abager@uol.com.br
}

\begin{abstract}
Fagundes, C.M. \& Bager, A. Reproductive ecology of Hydromedusa tectifera (Testudines: Chelidae) in southern Brazil. Biota Neotrop. May/Aug 2007 vol. 7, no. 2. http://www.biotaneotropica.org.br/v7n2/pt/ abstract?article+bn04807022007. ISSN 1676-0603.

The few available information on the reproductive behavior of Hydromedusa tectifera (Chelidae) are based on captive individuals. The H. tectifera reproductive biology was verified from 1996 to 2002 by non-systematic surveys in the Estação Ecológica do Taim, southern Brazil. Forty-six intact and damaged nests of the species were found. Mean clutch size was 11.6 eggs which exhibited a mean size of $36.5 \times 23.9 \mathrm{~mm}$ and an average weight of $11.5 \mathrm{~g}$. Individual mean eggs volume resulted an average of $10148.31 \mathrm{~mm}^{3}$. Nests had an average depth of 11.1 and $5.8 \mathrm{~cm}$ from the surface to the first egg. The eggs volume variation in the same nest was smaller $(\mathrm{CV}=4.71 \%)$ than among different nests $(\mathrm{CV}=12.32 \%)$. The eggs width was positively dependent of their length. In the same hatch the number of eggs did not depended of their size or volume. Nesting behavior was observed in November and December. The species showed higher frequency for nesting between 6 and 8 P.M., in areas with certain inclination of the substratum, between $20^{\circ}$ and $30^{\circ}$ of declivity. Higher nest abundance was verified up to $5 \mathrm{~m}$ from the lagoon. The results show that the data obtained for H. tectifera are similar to those previously reported for Australian snake-necked turtles and for species of Chelidae from southern Brazil.

Keywords: South American snake-necked turtle, nesting site, eggs, nests.

\section{Resumo}

Fagundes, C.M. \& Bager, A. Ecologia reprodutiva de Hydromedusa tectifera (Testudines: Chelidae) no sul do Brasil. Biota Neotrop. May/Aug 2007 vol. 7, no. 2. http://www.biotaneotropica.org.br/v7n2/pt/abstract? article+bn04807022007. ISSN 1676-0603.

Existem poucos dados na literatura sobre a reprodução de Hydromedusa tectifera (Chelidae), e estes se referem a indivíduos de cativeiro. A ecologia reprodutiva da espécie foi verificada através de monitoramentos não sistemáticos entre 1996 e 2002 na Estação Ecológica do Taim, no extremo sul do Brasil, com a intenção de reunir informações sobre a reprodução da espécie em ambiente natural. Foram encontrados 46 ninhos da espécie, entre intactos e predados. As ninhadas tiveram, em média, 11,6 ovos que apresentaram um tamanho médio de $36,5 \times 23,9 \mathrm{~mm}$. A média do peso dos ovos foi de $11,1 \mathrm{~g}$ e o seu volume individual resultou em uma média de $10148,31 \mathrm{~mm}^{3}$. Os ninhos alcançaram uma profundidade média de 11,1 e 5,8 $\mathrm{cm}$ da superfície ao primeiro ovo. A variação do volume dos ovos de um mesmo ninho foi menor $(\mathrm{CV}=4,71 \%)$ do que entre diferentes ninhos $(\mathrm{CV}=12,32 \%)$. A largura dos ovos foi positivamente dependente do seu comprimento. Já em uma mesma ninhada, o número de ovos não dependeu do seu tamanho e do seu volume. Foram observadas desovas nos meses de novembro e dezembro, mas a maioria delas ocorreu na segunda quinzena de novembro. A espécie desovou com maior frequiência no período das 18 às 20 horas, e em áreas com certa inclinação do substrato, entre $20^{\circ}$ e $30^{\circ}$ de declividade. A maior abundância de ninhos foi observada até $5 \mathrm{~m}$ de distância da lagoa. Os resultados encontrados demonstram congruência entre os dados obtidos para $H$. tectifera e aqueles existentes para cágados-de-pescoço-comprido da Austrália e para as espécies de Chelidae do extremo sul do Brasil.
\end{abstract}

Palavras-chave: cágado-de-pescoço-comprido, sítio de desova, ovos, ninhos. 


\section{Introdução}

A família Chelidae é composta por gêneros que habitam somente a América do Sul e a Australásia (Greer 2003). Nesta família estão inseridos os cágados-de-pescoço-comprido, sendo que Chelodina, da Austrália, é o gênero com maior número de espécies. Na América do Sul estes animais são representados pelo gênero Hydromedusa, que é composto por apenas duas espécies, H. maximiliani (Mikan 1820) e $H$. tectifera Cope, 1869. A primeira é endêmica das regiões montanhosas do sul da Bahia e sudeste do Brasil, com ocorrência nos estados de São Paulo, Rio de Janeiro, Minas Gerais e Espírito Santo (Souza 2005), enquanto que a segunda apresenta uma distribuição mais ampla, ocorrendo no Brasil meridional, Paraguai, Uruguai, Argentina e extremo sudeste da Bolívia (Lema \& Ferreira 1990). No Brasil, exemplos típicos são os cágados, os quais constituem o grupo com maior número de espécies de quelônios (Souza 2004). Além de $H$. tectifera, no extremo sul do país são encontradas outras duas espécies de Chelidae: Acanthochelys spixii e Phrynops hilarii (Lema \& Ferreira 1990).

O conhecimento da biologia e ecologia de Hydromedusa é escasso. Aspectos sobre a estrutura populacional, padrões de atividade (Souza \& Abe 1997, Souza 2004), habitat (Guix et al. 1992, Iverson 1992, Souza 2004), dieta (Guix et al. 1992, Souza \& Abe 1995, Souza 2004) e genética de H. maximiliani têm sido explorados (Souza et al. 2002a,b). No entanto, apesar das evidências sugeridas por Souza (2005) de que a espécie nidifica entre dezembro e janeiro, a estação reprodutiva de $H$. maximiliani ainda não foi descrita. Há também poucas informações a respeito dos ninhos e comportamento de desova da espécie (Guix et al. 1992, Souza 2004, 2005, Souza et al. 2006). Menos ainda é conhecido sobre H. tectifera (Souza 2004, Souza et al. 2006). Lema \& Ferreira (1990) relatam que a espécie vive em águas lênticas e permanece enterrada na lama no inverno, ressurgindo apenas na primavera. Benefield (1979) acompanhou o comportamento de corte, a desova e o nascimento de filhotes em cativeiro de uma única fêmea de H. tectifera. Já Lema \& Ferreira (1990) observaram desovas da espécie em um viveiro. Outros estudos em cativeiro indicaram o período de desova e características de nidificação (Freiberg 1981, Molina et al. 1998, Cabrera 1998, Métrailler 2000, Chinen et al. 2004, Souza et al. 2006). No entanto, trabalhos sobre a reprodução da espécie em ambientes naturais são inexistentes. Assim, neste estudo reunimos as primeiras informações acerca das características reprodutivas de H. tectifera em vida livre. Além disso, pretendeu-se identificar a relação da espécie com os Chelidae que habitam o extremo sul do país e com os cágados-de-pescoço-comprido da Austrália.

\section{Material e Métodos}

\section{1. Área de estudo}

O trabalho foi desenvolvido em duas localidades na Estação Ecológica do Taim (ESEC Taim) (Figura 1), uma localizada na margem oeste da Lagoa Mangueira, no limite sul da ESEC Taim (UTM 22H x = $0345990 \mathrm{mE}$; $\mathrm{y}=6364355 \mathrm{mN}$ ) e outra associada ao setor onde a ESEC Taim é segmentada por uma rodovia (BR-471) (UTM 22H x = $0353814 \mathrm{mE}$; $\mathrm{y}=6395605 \mathrm{mN}$ ).

A Estação Ecológica do Taim situa-se na planície costeira do Rio Grande do Sul. As formações sedimentares desta região encontram-se muito bem conservadas, e estão ocupadas por dezenas de lagunas de tamanhos variados que possuem grande acúmulo de sedimentos continentais (Calliari 1998). A Planície Costeira do Estado do Rio Grande do Sul está inserida no maior complexo lacunar da América do Sul, constituído pela Laguna dos Patos, Lagoa Mirim e Mangueira. A fisionomia da região compreende

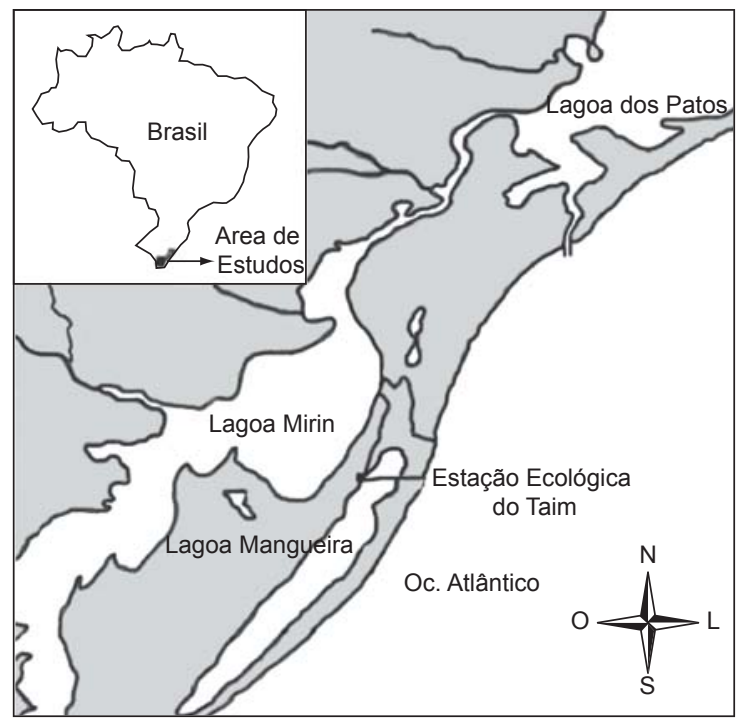

Figura 1. Área de estudo, mostrando o habitat aquático e a área de desova de Hydromedusa tectifera na ESEC Taim, RS, Brasil.

Figure 1. Study area, showing the aquatic habitat and nesting area of Hydromedusa tectifera in the ESEC Taim, RS, Brazil.

inúmeras lagoas costeiras associadas a banhados, matas paludosas e campos. O clima é do tipo subtropical úmido (Cfa, segundo Köeppen), caracterizado por chuvas bem distribuídas durante todos os meses (média anual de $1252 \mathrm{~mm}$ ). A temperatura do mês mais quente é superior a $22{ }^{\circ} \mathrm{C}$ e entre 3 e $18{ }^{\circ} \mathrm{C}$ no mês mais frio (Moreno 1961).

\section{Coleta e análise dos dados}

Os dados foram obtidos através de monitoramentos de campo não sistemáticos entre 1996 e 2002. Os ninhos (intactos ou não) foram identificados por procura ativa ou pelo encontro de fêmeas em processo de desova. No último caso, foi registrado o horário da desova. Os ovos foram contados, medidos com paquímetro de $0,1 \mathrm{~mm}$ de precisão e pesados com balança analítica com precisão de $0,1 \mathrm{~g}$, sendo devolvidos imediatamente ao ninho de origem. Ainda foram coletadas as informações da profundidade do ovo em relação à superfície e à profundidade total do ninho. $\mathrm{O}$ cálculo do volume dos ovos foi realizado através do grau de esfericidade e do formato do bicone (Maritz \& Douglas 1994).

A posição geográfica de cada ninho foi obtida com o auxílio de GPS. Os ninhos encontrados na Lagoa Mangueira no ano de 2001 e 2002 tiveram a sua distância até a margem, medida em linha reta, com auxílio de trena. Além disso, foi medida a declividade do solo no ponto onde o ninho foi construído. A relação entre o comprimento e a largura dos ovos, e entre estas medidas e o seu volume foi verificado através de regressão linear. Além disso, a análise de regressão foi utilizada na correlação entre o volume dos ovos e o número destes em cada ninho.

A variabilidade do volume dos ovos na mesma ninhada e entre ninhos diferentes foi avaliada pela comparação dos coeficientes de variação $(\mathrm{CV})$. Para determinar qual a declividade do substrato e a distância em relação à lagoa em que os ninhos tiveram maior ocorrência e a época e o horário em que as desovas foram mais freqüentes foi aplicado o teste do $\chi^{2}$. Todas as análises consideraram um nível de significância $\leq 0,05$. 


\section{Resultados}

Foram encontrados 46 ninhos de $H$. tectifera, considerando os intactos e os predados. Os ovos apresentaram casca calcárea, coloração branca e forma elíptica. As ninhadas tiveram em média ( \pm DP) $11,6 \pm 1,94$ ovos. A média do comprimento dos ovos foi $36,5 \pm 2,21 \mathrm{~mm}$, e a largura de 23,9 $\pm 1,1 \mathrm{~mm}$. O peso médio dos ovos foi $11,1 \pm 0,51 \mathrm{~g}$. A análise do volume dos ovos resultou em uma média de 10148,3 $\pm 1250,25 \mathrm{~mm}^{3}$ (Tabela 1). Houve uma menor variação do volume dos ovos de um mesmo ninho (CV=4,71\%), do que entre os diferentes ninhos $(\mathrm{CV}=12,32 \%)$.

O comprimento dos ovos de $H$. tectifera na ESEC Taim mostrou-se dependente da largura destes $\left(\mathrm{F}_{1,155}=52,83 ; \mathrm{P}<0,001\right)$. Porém, o número de ovos de cada postura não foi dependente do seu comprimento $\left(\mathrm{F}_{1,12}=0,01 ; \mathrm{P}=0,914\right)$, e da sua largura $\left(\mathrm{F}_{1,12}=2,24 ; \mathrm{P}=0,160\right)$. O volume dos ovos também não apresentou relação com o número de ovos em cada ninhada $\left(\mathrm{F}_{1,13}=0,65\right.$; $\mathrm{P}=0,562$ ) (Tabela 2). Em relação aos ninhos, estes apresentaram em média 11,1 $\pm 2,19 \mathrm{~cm}$ de profundidade total, e 5,8 $\pm 3,21 \mathrm{~cm}$ da superfície ao primeiro ovo.

As desovas foram observadas nos meses de novembro e dezembro, porém $77,3 \%$ das fêmeas em processo de nidificação foram encontradas na segunda quinzena de novembro $\left(n=34 ; \chi^{2}=92,36\right.$; g.l. $=4 ; \mathrm{P}<0,001)$. Foram verificados indivíduos nidificando pela manhã (8:46 horas), e à noite ( 23 horas). No entanto, as desovas da espécie foram mais freqüentes entre $18 \mathrm{e}$ 20 horas, quando $38,1 \%$ das posturas ocorreram $\left(n=9 ; \chi^{2}=29,14\right.$; g.l. = 8; P $<0,001$ ) (Tabela 3).

Apesar da declividade dos locais em que os ninhos foram depositados oscilarem entre $0^{\circ}$ e $55^{\circ}\left(\right.$ média $\left.=26,17^{\circ}\right)$, foi registrada maior abundância de ninhos em áreas inclinadas. Assim, 40,62\% dos ninhos foram encontrados em substratos apresentando entre $20^{\circ}$ e $30^{\circ}$ de declividade $(\mathrm{n}=13 ; \chi 2=26,19 ;$ g.l. $=6$; $\mathrm{P}<0,001)$ (Figura 2). A distância em linha reta dos ninhos em relação ao recurso hídrico variou entre 0,63 e 20,6 m (média = 8,2 m). Contudo,
$37,5 \%(\mathrm{n}=12)$ das desovas ocorreram até $5 \mathrm{~m}$ da lagoa $\left(\chi^{2}=14,563\right.$; g.l. $=4, \mathrm{P}=0,0057)$.

\section{Discussão}

No presente estudo, o tamanho da ninhada oscilou de sete a 15 ovos, assim como observado por Freiberg (1981) e Molina et al. (1998). Benefield (1979), por sua vez, registrou uma postura de 14 ovos. Entretanto, Chinen et al. (2004) no estado de São Paulo, identificaram uma média de 9,9 ovos por postura, menor que a encontrada neste trabalho (11,6 ovos). Resultados obtidos por outros autores, também em latitudes mais baixas em relação a este estudo, revelaram menor amplitude no número de ovos por ninhada (cinco a 10 ovos) (Cabrera 1998, Métrailler 2000). Conforme Saidler (1973), o tamanho médio das ninhadas tende a aumentar em altas latitudes, podendo ser essa uma hipótese para explicar a diferença constatada. No entanto, para animais de cativeiro, Lema \& Ferreira (1990) e A. Bager (dados não publicados) encontraram, respectivamente, ninhadas menores (4 a 6 ovos) e maiores (20 ovos) na mesma área geográfica. Tais modificações significantes nos padrões reprodutivos em populações próximas pode ser resultado do alimento disponível em cada ambiente (Moll \& Legler 1971). Animais em cativeiro estão expostos a condições distintas da natureza, principalmente com relação a sua

Tabela 3. Horário de desova, declividade do substrato e distância dos ninhos de Hydromedusa tectifera à margem da lagoa Mangueira na ESEC Taim, RS, Brasil.

Table 3. Nesting period, substrate declivity and straight-line distance of nests of Hydromedusa tectifera from the Mangueira Lagoon in the ESEC Taim, RS, Brazil.

\begin{tabular}{lrrccc}
\hline & $\bar{\chi}$ & \multicolumn{1}{c}{ DP } & Mín. & Máx. & $\mathrm{n}$ \\
\hline Distância da água (m) & 8,23 & 5,64 & 0,63 & 20,60 & 32 \\
Declividade (graus) & 26,20 & 12,40 & 0 & 55 & 32 \\
Horário (h) & $17: 52$ & $4: 15$ & $08: 46$ & $23: 00$ & 21 \\
\hline
\end{tabular}

Tabela 1. Caracterização dos ninhos e dos ovos de Hydromedusa tectifera, na ESEC Taim, RS, Brasil.

Table 1. Clutch, eggs, and nest characteristics of Hydromedusa tectifera, in the ESEC Taim, RS, Brazil.

\begin{tabular}{|c|c|c|c|c|c|c|}
\hline & $\bar{\chi}$ & DP & Mín. & Máx. & n ovos & $\mathrm{n}$ ninhos \\
\hline Número de ovos & 11,6 & 1,94 & 7 & 15 & - & 13 \\
\hline Largura dos ovos (mm) & 23,9 & 1,10 & 20,7 & 26,1 & 163 & 15 \\
\hline Comprimento dos ovos (mm) & 36,5 & 2,21 & 30,2 & 43,0 & 162 & 15 \\
\hline Peso dos ovos (g) & 11,1 & 0,51 & 10,3 & 11,9 & 12 & 1 \\
\hline Volume dos ovos $\left(\mathrm{mm}^{3}\right)$ & 10148,3 & 1250,25 & 7210,0 & 12602,2 & 126 & 15 \\
\hline Profundidade total do ninho $(\mathrm{cm})$ & 11,1 & 2,2 & 8,0 & 14,0 & - & 5 \\
\hline Profundidade do ninho até o $1^{\circ}$ ovo $(\mathrm{cm})$ & 5,8 & 3,2 & 1,5 & 8,5 & - & 4 \\
\hline
\end{tabular}

Tabela 2. Análise da regressão linear entre o número de ovos e o tamanho e o volume dos ovos e entre o comprimento dos ovos e a largura destes em uma mesma ninhada de Hydromedusa tectifera na ESEC Taim, RS, Brasil.

Table 2. Analysis of linear regression between number of the eggs and eggs size and egg volume and between egg length and egg width in the same clutch of Hydromedusa tectifera in the ESEC Taim, RS, Brazil. $\mathrm{n}=$ número de ninhos analisados.

\begin{tabular}{|c|c|c|c|c|c|c|}
\hline & \multicolumn{6}{|c|}{ Parâmetros da regressão } \\
\hline & $\mathbf{n}$ & $\mathbf{a}$ & $\mathbf{b}$ & $\mathbf{r}^{2}$ & $\mathbf{F}$ & $\mathbf{P}$ \\
\hline $\mathrm{N}^{\circ}$ de ovos $\mathrm{x}$ Comprimento dos ovos & 14 & $-0,02$ & 36,89 & 0,001 & 0,01 & $>0,05$ \\
\hline $\mathrm{N}^{\circ}$ de ovos $\mathrm{x}$ Largura dos ovos & 14 & 0,14 & 22,31 & 0,16 & 2,24 & $>0,05$ \\
\hline $\mathrm{N}^{\circ}$ de ovos $\mathrm{x}$ Volume dos ovos & 14 & 4,27 & 0,0006 & 4,81 & 0,65 & $>0,05$ \\
\hline Largura $\mathbf{x}$ comprimento dos ovos & 14 & 10,68 & 1,07 & 24,9 & 52,83 & $<0,05$ \\
\hline
\end{tabular}



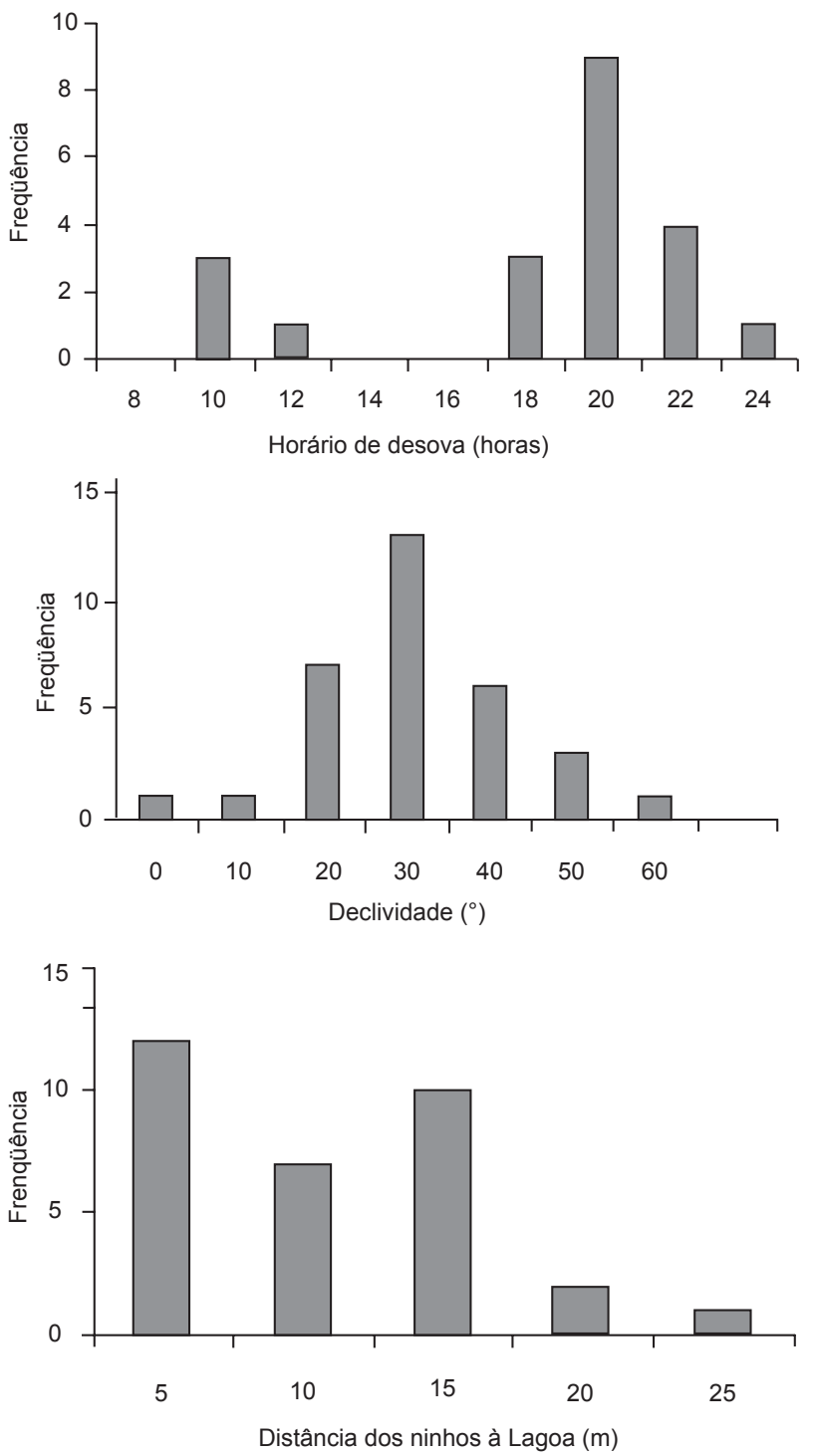

Figura 2. Frequiência do horário de desova, da declividade dos ninhos e da distância em linha reta dos ninhos de Hydromedusa tectifera à lagoa Mangueira na ESEC Taim, RS, Brasil.

Figure 2. Frequency of nesting schedule, nest declivity and the straight-line distance of nests of Hydromedusa tectifera from the Mangueira Lagoon in the ESEC Taim, RS, Brazil.

dieta. Assim, esta variação do padrão de alimentação pode alterar as reservas energéticas disponíveis para a reprodução, resultando em ninhadas maiores ou menores (Moll \& Legler 1971, Lindeman 1996).

Em geral, em quelônios, a relação entre o tamanho da fêmea e número de ovos por ninho é direta, ou seja, espécies de maior porte tendem a ter ovos maiores (Moll \& Legler 1971). A maioria das espécies de Chelidae que habitam o Brasil também confirma esta relação (Souza 2004). Hydromedusa tectifera alcança no máximo $30 \mathrm{~cm}$ de comprimento da carapaça (Ernst \& Barbour 1989). Nesse sentido, para $P$. hilarii, que atinge tamanho maior que a espécie de estudo, forma registradas ninhadas com valores médios de 14,2 ovos (Souza et al. 2006). Porém, em A spixii cujo tamanho é menor que $H$. tectifera, foram identificadas ninhadas menores (média $=4,4$ ovos) (Souza et al. 2006). Nos cágados-de-pescoço-comprido que exibem tamanho similar à espécie investigada neste trabalho, Chelodina oblonga e $C$. rugosa, verificaram-se ninhadas com variações mais parecidas a esta, de três a 16 ovos e de sete a 19 ovos, respectivamente (Greer 2003). A semelhança do tamanho da ninhada entre espécies com tamanho corporal parecido reforça a idéia de que o espaço de sua cavidade corporal exerce restrições quanto ao número de ovos produzidos (Congdon et al. 1983, Iverson \& Ewert 1991).

Em média, os ovos de $H$. tectifera examinados por Chinen et al. (2004) foram maiores que os aqui encontrados, tanto no comprimento (média $=41,5 \mathrm{~mm}$ ), como na largura (média $=25,8 \mathrm{~mm}$ ). Benefield (1979) identificou variação do comprimento dos ovos de 34 a $39 \mathrm{~mm}$, sendo o limite superior menor que o verificado neste trabalho. Já a média das medidas dos ovos observadas por Cabrera (1998) na Argentina foram muito parecidas com as apresentadas aqui. $\mathrm{O}$ fato das medidas dos ovos terem sido em média maior no estudo de Chinen et al. (2004) pode ser atribuída a existência da relação inversa entre latitude e tamanho dos ovos, que em geral ocorre em quelônios de água doce (Moll \& Legler 1971, Gibbons \& Greene 1990). O mesmo foi observado para $H$. maximiliani, que apesar de ser menor que a espécie de estudo, alcançando no máximo $19,8 \mathrm{~cm}$ de comprimento máximo da carapaça (Souza 2004), apresenta medidas dos ovos acima da média identificada aqui para $H$. tectifera. Naquela espécie, Yamashita (1990) verificou ovos de $40 \mathrm{~mm}$ de comprimento e $25 \mathrm{~mm}$ de largura, oriundos de uma fêmea em cativeiro, que foi trazida grávida de ambiente natural. Outro fator que contribui para a variação das medidas dos ovos entre populações da mesma espécie e entre espécie aparentadas diferentes é o tamanho corporal médio das fêmeas reprodutivas. Dessa forma, a qualidade do habitat e a disponibilidade de recursos podem ser responsáveis por estas diferenças na obtenção de tamanho (Gibbons \& Greene 1990). Acanthochelys spixii, espécie de pequeno porte, deposita ovos com diâmetros menores que H. tectifera, mostrando em média $26,5 \mathrm{~mm}$ de comprimento e 24,7 mm de largura (Souza et al. 2006). Com relação às espécies australianas, C. oblonga, é a espécie que mais se aproxima da espécie de estudo quanto ao tamanho dos ovos. Clay (1981) relatou uma média de $33,1 \mathrm{~mm}$ de comprimento e $23 \mathrm{~mm}$ de largura nos ovos da espécie.

Normalmente, em espécies de quelônios que exibem menores tamanhos, as fêmeas depositam maiores investimentos no tamanho dos ovos. Contudo, estas medidas são restringidas pela sua abertura pélvica, que acabam por limitar a sua largura. Já em espécies de maior tamanho, o aparato pélvico permitirá uma maior variação tanto no número quanto no tamanho dos ovos (Congdon et al. 1983, Iverson \& Ewert 1991). Tendo em consideração as medidas dos ovos verificadas por nós para $H$. tectifera, este modelo explica a média inferior do comprimento dos ovos $(32,6 \mathrm{~mm})$ encontrada por Souza et al. (2006) para $P$. hilarii. Da mesma forma, a média da largura dos ovos $(31,9 \mathrm{~mm})$ registrada por estes autores foi bem maior do que a média identificada aqui para $H$. tectifera.

Freiberg (1981) e Molina et al. (1998) encontraram ovos de H. tectifera pesando entre 10 e 22,5 g. Essa variação foi semelhante ao relatado por Chinen et al. (2004). Já a massa dos ovos analisados por Benefield (1979) variou entre 10 e 12,5 g, ficando mais próximo dos dados apresentados por nós. Porém, essa diferença em relação aos três primeiros autores pode refletir o baixo número de ovos pesados neste trabalho, sendo provenientes de apenas um ninho. Conforme constatado por Souza et al. (2006), as posturas de A. spixii e P. hilarii mostram ovos com, respectivamente, 9,6 e 19,9 g em média. Chelodina oblonga possui ovos com média de peso bastante parecida com a variação relatada para $H$. tectifera, possuindo segundo Clay (1981) média de 10,4 g. Assim, o peso dos ovos também parece apresentar relação com o tamanho atingido em cada espécie. Por outro lado, o cágado australiano $C$. longicollis, que alcança maior tamanho em comparação a $H$. tectifera, além de apresentar ovos menores que a espécie de estudo, também exibe média de peso bas- 
tante inferior, 7,5 g para Legler \& Cann (1980) e 6,4 g para Kennett \& Georges (1990). Nesta espécie, portanto, a seleção parece estar operando para produzir um tamanho ótimo de ninhada e não um tamanho ótimo dos ovos.

Houve uma relação positiva entre o comprimento e a largura dos ovos, provavelmente devido a sua largura ser limitada pela abertura da cintura pélvica. O tamanho e o volume dos ovos de $H$. tectifera não foram dependentes do número de ovos de cada ninhada. Assim, estas variáveis, como em outras espécies de quelônios, podem estar associadas ao tamanho das fêmeas (Congdon et al. 1983, Iverson 1991, Iverson \& Smith 1993).

Ninhos e fêmeas de $H$. tectifera em atividade de desova foram encontrados somente nos meses de novembro e dezembro. O período reprodutivo identificado neste trabalho foi menor do que o verificado em outros estudos da espécie sob diferentes regiões geográficas, onde a estação de desova aconteceu de novembro a maio (Freiberg 1981, Molina et al. 1998), e ainda, a partir do final de outubro ao início de abril, com $75 \%$ das posturas entre novembro e janeiro (Chinen et al. 2004). Na Argentina, Cabrera (1998) registrou desovas entre outubro e janeiro. Tais variações na temporada reprodutiva de $H$. tectifera podem estar relacionadas com o efeito das diferenças de temperatura do ambiente na fisiologia dos indivíduos. Em quelônios, o início da estação de desova varia de acordo com as temperaturas do início da primavera, coincidindo normalmente com temperaturas mais quentes do ar ou da água (Weishampel et al. 2004). Nesse sentido, climas tropicais permitem a ocorrência de períodos reprodutivos mais longos (Moll \& Moll 1990).

Souza \& Abe (1997) propuseram uma temporada similar de desova para $H$. maximiliani. Segundo esses autores a época de reprodução da espécie deve ocorrer entre dezembro e janeiro. No sul do Brasil, $P$. hilarii nidifica em dois períodos distintos, entre fevereiro e maio e entre setembro e dezembro (Souza et al. 2006), este último se sobrepondo a época de desova estipulada para $H$. tectifera. Em contrapartida, o período de nidificação de A. spixii inicia após a temporada reprodutiva da espécie de estudo, entre fevereiro e maio (Souza et al. 2006). Da mesma forma, o cágado-de-pescoço-comprido australiano $C$. longicollis faz suas posturas na primavera e início do verão, de novembro a janeiro, embora a maioria das desovas ocorra nos meses de novembro e dezembro (Greer 2003). Porém, C. expansa demonstra grande modificação no modelo típico de zona temperada, ovipositando no outono e no inverno (Legler \& Georges 1993).

Apesar de terem sido registradas fêmeas em atividade de nidificação tanto durante o dia quanto à noite, a maior ocorrência de desovas foi identificada do entardecer para a noite. Lema (1994) já havia ressaltado que a espécie, assim como A. spixii, é caracterizada pelos seus hábitos noturnos. Este comportamento, entretanto, é diferente do verificado para $P$. hilarii, onde as desovas são realizadas durante o dia, principalmente pela manhã (Bujes 1998). Hydromedusa tectifera difere também da espécie australiana $C$. expansa, onde as posturas acontecem no período diurno (Georges 1984). Já C. longicollis nidifica durante a tarde e a noite, sendo que as desovas durante o dia ocorrem sob condições de nebulosidade (Beck 1991). Outros estudos devem ser realizados a fim de verificar se as desovas diurnas de $H$. tectifera também estão relacionadas à baixa luminosidade.

A profundidade dos ninhos pode proporcionar diferenças de microambientes em seu interior (Ferreira \& Castro 2005), pois ovos em ninhos mais rasos ou no topo de ninhos são expostos a maior variação diária de temperatura (Congdon \& Gibbons 1990). Entre os Chelidae brasileiros, normalmente espécies de pequeno porte depositam seus ovos a profundidades muito pequenas (Souza 2004). Em contraste a este padrão, $H$. tectifera construiu seus ninhos a uma profundidade média de $11,1 \mathrm{~cm}$, próxima àquela estipulada para $P$. hilarii (média $=12,1 \mathrm{~cm}$ ) por Bujes (1998). Na maioria das espécies do gênero Chelodina, os ninhos geralmente atingem $15 \mathrm{~cm}$ (Greer 2003) (exceto para $C$. longicollis, onde foram identificados tanto ninhos mais rasos quanto mais profundos). Porém, Booth (2002) encontrou um ninho de $C$. expansa $\mathrm{com} 22 \mathrm{~cm}$ de profundidade, onde o primeiro ovo estava a $9 \mathrm{~cm}$ da superfície.

Conforme Souza (2004), os ninhos dos cágados brasileiros são construídos longe da água, sendo que as características ambientais que mais influenciam na escolha do local de nidificação são a cobertura vegetal, o tipo do substrato e a inclinação do terreno. De acordo com Clay (1981), C. oblonga, assim como $H$. tectifera, exibiu preferência por desovar em áreas com maior declividade do substrato. Em muitas populações de quelônios, a maior frequiência de ninhos depositados longe dos recursos hídricos pode ocorrer devido à predação ser extremamente alta em posturas perto da água (Burke et al. 1998). Phrynops hilarii nidifica a uma distância média de $80 \mathrm{~m}$ do leito d'água mais próximo (Bujes 1998). No entanto, a espécie de estudo, da mesma forma que $A$. spixii, exibiu maior abundância de ninhos em áreas próximas à água. Esta última faz suas posturas cerca de $10 \mathrm{~m}$ dos rios (D'Amato \& Morato 1991). H. tectifera apresentou semelhança com C. expansa, que também deposita seus ninhos com maior ocorrência nas proximidades de recursos hídricos. Contudo, foram observados ninhos da espécie australiana distando até $750 \mathrm{~m}$ do corpo d'água (Georges 1984). Já em C. longicollis as áreas de desovas variam de 1,5 a 500 m de distância da água (Greer 2003). Nestas espécies, a estratégia de ovipositar perto da água se deve ao maior risco de predação ocasionado pelo maior deslocamento da fêmea em terra (Congdon et al. 1983) e, principalmente em espécie que nidificam na primavera e no verão, ao maior risco de dessecação (Greer 2003).

Os resultados encontrados demonstram congruência entre os dados obtidos para $H$. tectifera e aqueles existentes para cágados-de-pescoço-comprido da Austrália e para as espécies de Chelidae do extremo sul do Brasil. Houve maior similaridade nas medidas e peso dos ovos e no tamanho das ninhadas entre espécies de porte parecido com $H$. tectifera. Nossos dados demonstram que $H$. tectifera é uma espécie suscetível ambientalmente, requerendo sítios de desova específicos (alta declividade e proximidade ao meio hídrico). Novas pesquisas poderão elucidar se existem outros parâmetros ambientais que condicionam a atividade reprodutiva da espécie. Também devem ser realizados esforços em estudos da ecologia reprodutiva, sobretudo sobre aspectos de predação de ninhos e sucesso reprodutivo, de forma a subsidiar a elaboração de modelos populacionais para a espécie.

\section{Agradecimentos}

Os autores agradecem aos colegas de laboratório que ajudaram nos trabalhos de campo. Os autores são gratos também a Dra. Sonia Zanini Cechin pelas suas sugestões na leitura do manuscrito.

\section{Referências Bibliográficas}

BECK, R.G. 1991. The common long-necked tortoise Chelodina longicollis (Shaw 1802) (Testudines: Chelidae): a comparative study of the morphology and behaviour of disjunct populations. S. Aust. Nat.66:4-21.

BENEFIELD, J. 1979. Hatching the Argentine snake-necked turtle Hydromedusa tectifera at San Antonio Zoo. Int. Zoo Yearb. 19:55-58.

BOOTH, D.T. 2002. The breaking of diapause in embryonic broad-shell river turtles (Chelodina expansa). J. Herpetol.36(2):304-307.

BUJES, C.S. 1998. Atividade de nidificação de Phrynops hilarii Duméril \& Bibron (Testudines, Chelidae) na Reserva Biológica do Lami, Rio Grande do Sul, Brasil. Rev. Bras. Zool.15(4):921-928. 
BURKE, V.J., RATHBUN, S.L., BODIE, J.R. \& GIBBONS, J.W. 1998. Effect of density on predation rate for turtle nests in a complex landscape. Oikos 83(1):3-11.

CABRERA, M.A. 1998. Las Tortugas Continentales da Sudamérica Austral. BR Cópias, Córdoba, Argentina.

CALLIARI, L.J. 1998. O ambiente e a biota do estuário da Lagoa dos Patos. In Os ecossistemas costeiro e marinho do extremo sul do Brasil (U. Seeliger, C. Odebrecht \& J.P. Castello, eds). Ecoscientia, Rio Grande, p.13-18.

CHINEN, S., LISBOA, C.S. \& MOLINA, F.B. 2004. Biologia reprodutiva de Hydromedusa tectifera em cativeiro (Testudines, Chelidae). Arq. Inst. Biol. 71:401-403.

CLAY, B.T. 1981. Observations on the breeding biology and behaviour of the long-necked tortoise, Chelodina oblonga. J. Roy. Soc. West. Australia 4:27-32.

CONGDON, J.D., TINKLE, D.W., BREITENBACH, G.L. \& VAN LOBEN SEALS, R.C. 1983. Nesting behavior and hatching success in the turtle Emydoidea blandingi. Herpetologica 39(4):417-429.

CONGDON, J.D. \& GIBBONS, J.W. 1990. Turtle Eggs: Their Ecology and Evolution. In Life History and Ecology of the Slider Turtle (J.W. Gibbons, ed.). Smithsonian Institution Press, Washington, DC, p.109-123.

D’AMATO, A.F. \& MORATO, S.A.A. 1991. Notas biológicas e localidades de registro de Platemys spixii (Duméril \& Bibron, 1835) (Testudines: Chelidae) para o resto do estado do Paraná, Brasil. Acta Biol. Leopol. 13:119-130.

ERNST, C.H. \& BARBOUR, R.W. 1989. Turtles of the World. Smithsonian Institution Press, Washington D.C.

FERREIRA, P.D. Jr. \& CASTRO, P.T. 2005. Nest placement of the giant Amazon River turtle, Podocnemis expansa, in the Araguaia River, Goiás state, Brazil. Ambio 34(3):212-217.

FREIBERG, M.A. 1981. Turtles of South America. Tropical Fish Hobyst, Neptune.

GEORGES, A. 1984. Observations on the nesting and natural incubation of the long-necked tortoise Chelodina expansa in south-east Queensland. Herpetofauna 15:27-31.

GREER, A.E. 2003. Encyclopedia of Australian Reptiles. Australian Museum Online. http://www.amonline.net.au/ (último acesso em: 09/01/2007)

GIBBONS, J.W. \& GREENE, J.L. 1990. Reproduction in the slider and other species. In Life History and Ecology of the Slider Turtle (J.W. Gibbons, ed.). Smithsonian Institution Press, Washington, DC, p.124-134.

GUIX, J.C., MIRANDA, J.R. \& NUNES, V.S. 1992. Observaciones sobre la ecologia de Hydromedusa maximiliani. Boletín de la Asociación Herpetológica Española,3:23-25.

IVERSON, J.B. 1991. Patterns of survivorship in turtles (order Testudines). Can. J. Zool69(2):385-391.

IVERSON, J.B. \& EWERT, M.A. 1991. Physical characteristics of reptilian eggs and a comparison with avian eggs. In Egg incubation: its effect on embryonic development in birds and reptiles (D.C. Deeming \& M.W. Ferguson, eds). Cambridge University Press, New York, p.88-100.

IVERSON, J.B. 1992. A Revised Checklist With Distribution Maps of the Turtles of the World. Privately printed. Richmond, Indiana.

IVERSON, J.B. \& SMITH, G.R. 1993. Reproductive ecology of the Painted Turtle (Chrysemys picta) in the Nebraska Sandhills and across its range. Copeia 1993:1-21.

KENNETT, R.M. \& GEORGES, A. 1990. Habitat utilization and its relationship to growth and reproduction of the eastern long-necked turtle, Chelodina longicollis (Testudinata: Chelidae) from Australia. Herpetologica 46(1):22-33.

LEGLER, J.M. \& CANN, J. 1980. A new genus and species of chelid turtle from Queensland, Australia. Contributions to Science of the Natural History Museum, Los Angeles 324:1-18.
LEGLER, J.M., \& GEORGES, A. 1993. Family Chelidae. In: Fauna of Australia. Amphibia \& Reptilia. (C.J. Glasby, G.J.B. Ross \& P.L. Beesley, eds). Australian Government Printing Service, Canberra, v. 2A, p.142-152.

LEMA, T. \& FERREIRA, M.T.S. 1990. Contribuição ao conhecimento dos Testudines do Rio Grande do Sul (Brasil) - Lista sistemática comentada (Reptilia). Acta Biol. Leopol.12(1):125-164.

LEMA, T. 1994. Lista comentada dos répteis ocorrentes no Rio Grande do Sul, Brasil. Comun. Mus. Cienc. Pucrs Ser. Zool.7:41-150.

LINDEMAN, P.V. 1996. Comparative life history of painted turtle (Chrysemys picta) in two habitats in the inland Pacific Northwest. Copeia 1996:114-130.

MARITZ, F.M. \& DOUGLAS, R.M. 1994. Shape quantization and the estimation of volume and surface area of reptile eggs. J. Herpetol. 28(3):281-291.

MÉTRAILLER, S. 2000. Note sur Hydromedusa tectifera Cope, 1869 en Argentine. Manouria 3(7):23-32.

MOLL, E.O. \& LEGLER, J.M. 1971. The life history of a neotropical slider turtle, Pseudemys scipta (Schoepff), in Panama. Bul. Los Angeles Co. Mus. Nat. Hist. Sci. 11:1-102.

MOLL, D \& MOLL, E.O. 1990. The Slider Turtle in the Neotropics: Adaptation of a Temperate Species to a Tropical Environment. In Life History and Ecology of the Slider Turtle (J.W. Gibbons, ed.). Smithsonian Institution Press, Washington, DC, p.152-161.

MOLINA, F.B., ROCHA, M.B. \& LULA, L.A.B.M. 1998. Observações sobre o comportamento alimentar e a dieta de Phrynops hilarii em cativeiro (Reptilia, Testudines, Chelidae). Rev. Bras. Zool.15(1):73-79.

MORENO, J.A. 1961. Clima do Rio Grande do Sul. Secretaria da Agricultura, Porto Alegre.

SAIDLER, R. 1973. The reproduction of vertebrates. Academic Press, New York.

SOUZA, F.L. \& ABE, A.S. 1995. Observations on feeding habits of Hydromedusa maximiliani (Testudines, Chelidae) in southeastern Brazil. Chel. Conserv. Biol.1(4):320-322.

SOUZA, F.L. \& ABE, A.S. 1997. Population structure, activity, and conservation of the neotropical freshwater turtle, Hydromedusa maximiliani, in Brazil. Chel. Conserv. Biol.2(4):521-525.

SOUZA, F.L., CUNHA, A.F., OLIVEIRA, M.A., PEREIRA, G.A.G., PINHEIRO, H.P. \& REIS, S.F. dos. 2002a. Partitioning of molecular variation at local spatial scales in the vulnerable neotropical freshwater turtle, Hydromedusa maximiliani (Testudines, Chelidae): implications for the conservation of aquatic organisms in natural hierarchical systems. Biol. Conserv.104:119-126.

SOUZA, F.L., CUNHA, A.F., OLIVEIRA, M.A., PEREIRA, G.A.G. \& REIS, S.F. dos.2002b. Estimating dispersal and gene flow in the neotropical freshwater turtle Hydromedusa maximiliani (Chelidae) by combining ecological and genetic methods. Genet. Mol. Biol.25(2):151-155.

SOUZA, F.L. 2004. Uma revisão sobre padrões de atividades reprodutivas e alimentação de cágados brasileiros (Testudines, Chelidae). Phyllomedusa 3(1):15-27.

SOUZA, F.L. 2005. The Brazilian Snake-Necked Turtle. Reptilia 40:47-51.

SOUZA, F.L., GIRARDELLI, G.R. \& MARTINS, T.A. 2006. Reproductive aspects of Brazilian side-necked turtles (Chelidae). Bol. Asoc. Herpetol. Esp.17:28-34.

WEISHAMPEL, J.F., BAGLEY, D.AA. \& EHRHART, L.M. 2004. Earlier nesting by loggerhead sea turtles following sea surface warming. Glob. Change Biol.10:1424-1427.

YAMASHITA, C. 1990. Hydromedusa maximiliani ecology. Herp. Review. 21(1):1-19. 